\title{
Effect of Electrode Surface Modification by Sulfide on QCM Based Protein Biosensor
}

\author{
Yu-Cheng Lin ${ }^{1}$, Yi-Chi Chen ${ }^{1}$, Liang-Yu Chen ${ }^{2}$ \\ ${ }^{1}$ Department and Graduate Institute of Electronic Eng, Ming Chung University, Taoyuan, Chinese Taipei \\ ${ }^{2}$ Department and Graduate Institute of Biological Technol, Ming Chung University, Taoyuan, Chinese Taipei \\ Email: yclin@mail.mcu.edu.tw, ioknath@mail.mcu.edu.tw
}

Received 2013

\begin{abstract}
The rapid development of surface sensitive biosensor technologies requires optimum control of surface modification to provide reliable and reproducible results. With the aim to assemble a quartz crystal microbalance (QCM)-based protein biosensor, we focus our attention on sulfide receptor and its integration with the surface of the electrode. Here, we present different surface modification processing time to allow sulfide molecules to be immobilized to gold coated sensor for QCM sensing. The optimum surface modification processing time is also obtained by bovine serum albumin (BSA) binding measurement.
\end{abstract}

Keywords: Biosensor; QCM; Protein Binding; Surface Modification

\section{Introduction}

An important advance in biosensor was done during the last two decades. Especially, protein biosensors are now intensely developed for diagnostic applications [1], environmental monitoring [2] and food controls [3]. Masssensitive biosensor systems have attracted considerable attention in recent years since many important physical and chemical processes can be followed by observing the associated mass changes. The QCM system is based on the principle that the resonant frequency shifts of a piezoelectric crystal are directly proportional to the adsorbed mass [4]. QCM is suitable for several applications especially in biosensors [5]. QCM biosensors are time resolved, enough sensitive to detect non-labeled DNA [6], enough selective to detect single mismatch DNA [7], and renewable [3]. QCM protein biosensors may be designed by a wide choice of immobilization techniques of short receptor on the quartz microbalance electrode surface. As immobilization techniques of the protein probe on the surface are essential and critical for biosensor, we evaluate in this work different receptor immobilization time with adsorption of a disulfide on a gold-covered quartz surface of a microbalance. The disulfide immobilization process has several advantages: the adsorption of the receptor by binding between sulfur atom of disulfide and a gold atom of the surface is irreversible and stable. It takes less than one hour [8] and gold is very stable versus oxidation. And then, BSA is used to verify the sensing processing and to obtain the optimum receptor immobi- lization time.

\section{Operation Principle of QCM}

A flat quartz disc with electrodes on both surfaces can be forced to oscillate in a transverse acoustic mode (motion parallel to the surface) by an RF voltage applied at the acoustical resonance frequency of the plate. This device is called a transverse shear mode (TSM) quartz plate resonator. The frequency of the fundamental mode is inversely proportional to plate thickness and mass. TSM quartz plate resonators have been used as sensitive microbalances for thin adherent films since the late 1950 's, following the pioneering work of Sauerbrey [9]. The frequency of TSM resonator is continuously monitored when a sample is deposited on its surface. The shift in frequency due to deposition of a film of the same acoustic impedance as quartz is proportional to the deposited mass per unit area of the film,

$$
\Delta f=\frac{-2 f_{0}^{2}}{A \sqrt{\rho_{q} \mu_{q}}} \Delta m
$$

where $\mu_{\mathrm{q}}, \rho_{\mathrm{q}}$ and $\mathrm{f}_{0}$ are the shear modulus, density and the resonant frequency of bare quartz crystal and $\mathrm{A}$ and $\Delta \mathrm{m}$ are the electrode area and sampling mass difference, respectively. QCMs have been used as film thickness monitors in vacuum deposition of metals and inorganic solids. QCMs are useful because of their sub-nanogram sensitivity. 


\section{Experiment}

\subsection{QCM Apparatus}

The microbalance resonators, provided by Ya-Shin company, are AT-cut planar quartz crystals, $7 \mathrm{~mm}$ in diameter, with a $9 \mathrm{M} \mathrm{Hz}$ nominal resonance frequency. Two identical gold electrodes, $500 \AA$ in thickness and 3.5 $\mathrm{mm}$ in diameter, were deposited on both sides of the quartz. The QCM chip structure is shown in Figure 1. The crystal is mounted between two O-ring seals inserted in a glass cell to form an experimental cell. The gold side of the quartz used in the experiments wass cleaned with a pickling solution for 5 minutes, the sulfuric acid and hydrogen peroxide in a 1:3 ratio, and then rinsed with deionized water. We use 4,4'-dithiodibutyric acid to modify electrode surface. First, the sulfide powder was diluted with aqueous ethanol. The concentration of sulfide solution was 500 ppm in the experiment. Subsequently, sulfide solution was dropped on four QCM chip surface full and lasting for $15,30,45$ and 60 minutes at $25^{\circ} \mathrm{C}$, respectively, to immobilize protein receptor on gold electrode surface

\subsection{Protein Detection}

The ADS Plus instrument offers a platform to measure equilibrium binding affinity and kinectics using unmodified molecules in solution phase. The system allowing affinity measurement when reach equilibrium and kinetics measurement under pre-equilibrium conditions was used in our experiment. QCM chip was inserted into ADS PLUS and a computer was connected to the instrument for data acquisition. Deionized water or BSA solution via a peristaltic pump sent to the QCM chip, the system architecture is shown in Figure 2.

Initially, the average resonant frequency of the deionized water on QCM was recorded for 10 minutes after stable state. The stable frequency is as a benchmark. Then, a BSA solution of $50 \mathrm{ppm}$ was injected through

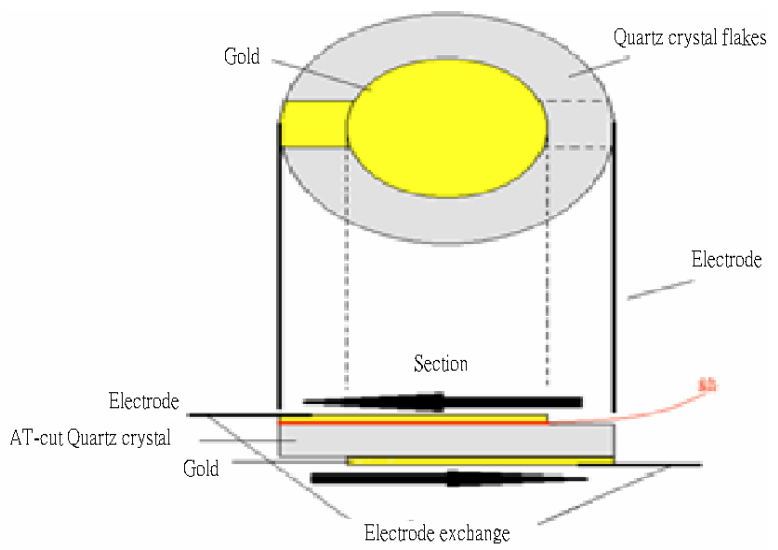

Figure 1. QCM chip structure. peristaltic pump. In order to observe the BSA binding kinetics and find the binding time, we use a QCM chip with 60 minutes sulfide modification to monitor the binding process for a long time. In terms of the QCM resonant frequency variation, we could observe the BSA binding saturation time. The injection process has three periods. The first one is 1600 seconds for water, the second one is 7200 seconds for BSA solution of $50 \mathrm{ppm}$ and the last one is 1800 seconds for water again. The QCM resonant frequency during injection process is shown in Figure 3. After injection BSA about 1800 seconds, which occurred at 3400 seconds in Figure 3, the resonant frequency largely degraded to $-3093 \mathrm{~Hz}$. Approximately after 10,400 seconds, the resonant frequency was almost stable. In other words, the BSA binding was completed and saturated. Then, deionized water was injected again to verify BSA strong binding. There was only slight increase in frequency owing to some BSA molecule weak binding escape. Since most of the sulfides for protein binding strength are very strong, it is belong to strong binding. For the binding time observation, we could fine that $86 \%$ frequency degradation occurred at about 30 minutes after BSA injection. So, the BSA binding time is set as 30 minutes (1800 seconds) for our work.

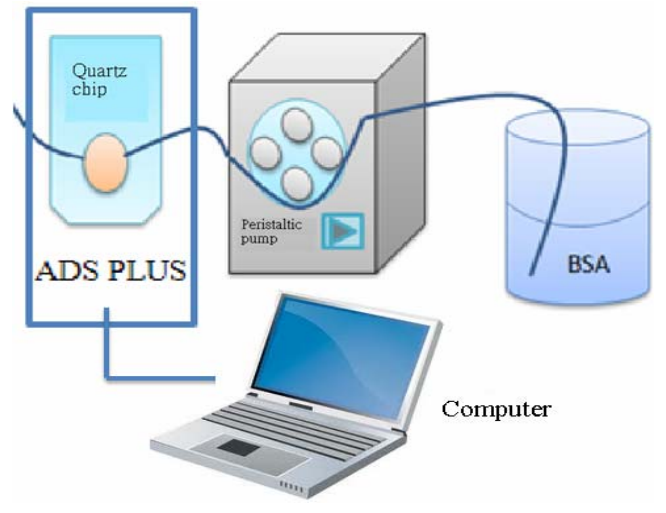

Figure 2. ADS PLUS and QCM for BSA binding detection.

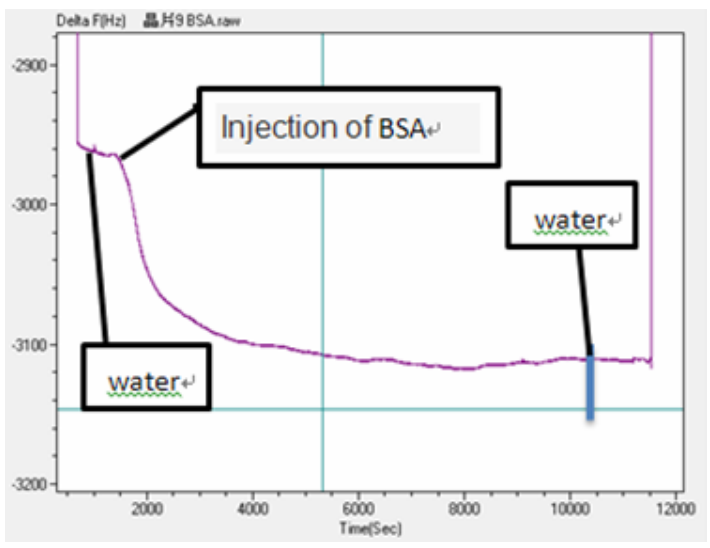

Figure 3. Resonant frequency variation during protein binding process. 
Table 1. Surface modification and protein binding effect.

\begin{tabular}{lcccc}
\hline \multicolumn{1}{c}{ chip } & \# 1 & \# 2 & \# 3 & \# 4 \\
\hline Surface modification time (minute) & 15 & 30 & 45 & 60 \\
BSA binding time (minute) & 30 & 30 & 30 & 30 \\
BSA frequency shift (Hz) & 117.3 & 136.8 & 137.8 & 138.0 \\
Efficiency (\%) & 3.75 & 4.37 & 4.35 & 4.65 \\
Adsorption mass (ng) & 314 & 366 & 368 & 369 \\
\hline
\end{tabular}

\section{Results and Discussion}

The frequency difference after BSA injection for 4 QCM chips with modification time of 15, 30, 45 and 60 minutes, respectively, is list in Table 1. Since sulfide could bind BSA and adsorb the mass on the surface of the chip, the resonant frequency would degrade. More BSA be binded, larger frequency difference will be. The frequency difference were measured as $117.3 \mathrm{~Hz}, 136.8 \mathrm{~Hz}$, $137.8 \mathrm{~Hz}$ and $138.0 \mathrm{~Hz}$ for the $15,30,45$ and 60 minutes, respectively. The efficiency is defined as the frequency difference divided with the initial deionized water resonant frequency. The results are $3.75 \%, 4.37 \%, 4.35 \%$ and $4.65 \%$, respectively. So, the optimum sulfide modification time for BSA protein QCM biosensor is about 30 minutes.

\section{Conclusions}

In this study, we present different surface modification processing time to allow sulfide molecules to be immobilized to golden electrode for QCM protein sensor. The surface modification was also tested by BSA binding measurement. The optimum surface modification processing time is detected as 30 minutes.

\section{REFERENCES}

[1] M. Campàs and I. Katakis, "DNA Biochip Arraying, Detection and Amplification Strategies Trend," Analytical Chemistry, Vol. 23, 2004, pp. 49-62.

[2] S. Rodriguez-Mozaz, M. J. López de Alda, M.-P. Marco and D. Barceló, "Biosensors for Environmental Monitoring A Global Perspective," Talanta, Vol. 65, 2005, pp. 291-297, "Title of Paper If Known," unpublished. doi:10.1016/S0039-9140(04)00381-9

[3] I. Mannelli, M. Minunni, S. Tombelli and M. Mascini "Quartz Crystal Microbalance (QCM) Affinity Biosensor for Genetically Modified Organism (GMOs) Detection," Biosensors and Bioelectronics, Vol. 18, 2003, pp. 129-140.doi:10.1016/S0956-5663(02)00166-5

[4] J. Rickert, A. Brecht and W. Gopel, "Quartz Crystal Microbalances for Quantitative Biosensing and Characterizing Protein Multilayers," Biosensors and Bioelectronics, Vol.12, pp. 567-575, Mill Valley, CA: University Science, 1989.

[5] S. Lin, C. C. Lu, H. F. Chien and S. M. Hsu. "An On-line Quantitative Immunoassay System Based on A Quartz Crystal Crobalance," Journal of Immunological Methods, Vol. 239, No.1-2, 2000, pp. 121-124. doi:10.1016/S0022-1759(00)00184-8

[6] Y. Okahata, Y. Matsunobu, K. Ijiro, M. Mukae, A. Murakami and K. Makino, "Hybridization of Nucleic Acids Immobilized on A Quartz Crystal Microbalance, Journal of the American Chemical Society,Vol.114,1992,pp. 8299-8300.doi:10.1021/ja00047a056

[7] F. Höök, A. Ray, B. Nordén and B. Kasemo, "Characterization of PNA and DNA Immobilization and Subsequent Hybridization with DNA Using Acoustic-Shear-Wave Attenuation Measurements," Langmuir, Vol. 17, 2001, pp. 8305-8312.doi:10.1021/la0107704

[8] E. Huang, M. Satjapipat, S. Han and F. F Zhou, "Surface Structure and Coverage of An Oligonucleotide Probe Tethered onto A Gold Substrate and Its Hybridization Efficiency for A Polynucleotide Target," Langmuir, Vol. 17 2001, pp. 1215-1224.doi:10.1021/la001019i

[9] G. Sauerbrey, Z. Physik, Vol. 155, 1959, p. 206.doi:10.1007/BF01337937 